\title{
RNA interference-mediated silencing of SOCS-1 via lentiviral vector promotes apoptosis of alveolar epithelial cells in vitro
}

\author{
YAN-RONG QIAN*, QIU-RUI ZHANG ${ }^{*}$, TING CHENG, HUAN-YING WAN and MIN ZHOU \\ Department of Respiratory Medicine, Ruijin Hospital, Affiliated to Shanghai Jiaotong University, Shanghai 20025, P.R. China
}

Received August 28, 2011; Accepted November 7, 2011

DOI: $10.3892 / \mathrm{mmr} .2011 .672$

\begin{abstract}
Suppressor of cytokine signaling-1 (SOCS1) is a protein that negatively regulates cytokine and growth factor signaling. However, little is known regarding the precise role it plays in idiopathic pulmonary fibrosis. The aim of the present study was to construct a recombinant lentiviral vector for RNA interference targeting the SOCS1 gene and to detect the expression in human alveolar epithelial cells. A lentiviral vector-mediated RNA interference method was used to establish a SOCS1-negative cell line of alveolar origin (A549). Three pairs of complementary small hairpin RNA (shRNA) oligonucleotides targeting the SOCS1 gene were designed, synthesized and inserted into the pPl13.7 vector. Packaged lentivirus particles were obtained after $48 \mathrm{~h}$, and the supernatant was used to transfect the human alveolar epithelial cell line A549. The expression of the SOCS1 protein was detected by Western blotting. MTT assay was used to detect the cell proliferation of alveolar epithelial cells with SOCS1 knockdown. The recombinant plasmids were confirmed by sequencing. The lentivirus-containing supernatant effectively infected the A549 cell line, and the expression of SOCS1 protein was inhibited, which was confirmed by Western blotting in the target cells. MTT assay indicated the inhibition effect for cell proliferation of A549 cells in the SOCS1-RNA interference group, compared to the control group with no interference-mediated silencing of the SOCS1 gene. A lentiviral vector for RNA interference targeting the SOCS1 gene was successfully constructed, and cell survival tests showed that knockdown of the SOCS1 gene promotes the apoptosis of alveolar cells.
\end{abstract}

Correspondence to: Dr Min Zhou, Department of Respiratory Medicine, Ruijin Hospital, Affiliated to Shanghai Jiaotong University, No. 197 Ruijin 2nd Road, Shanghai 20025, P.R. China

E-mail: doctor_zhou_99@yahoo.com.cn

*Contributed equally

Key words: lentiviral vector, RNA interference, suppressor of cytokine signaling-1, A549 cell line

\section{Introduction}

Suppressor of cytokine signaling (SOCS) is a family of proteins which negatively regulate cytokine and growth factor signaling. There are eight members and most of them take part in a negative feedback loop to attenuate cytokine signaling (1-3). Among them, SOCS1 was initially recognized as a repressor factor of IFN- $\gamma$, which is a critical molecule involved in the initiation of inflammation and is required for normal postnatal growth and survival (4-6). Knockout studies in mice suggest the role of SOCS1 as a modulator of IFN- $\gamma$ action (7). Further studies have shown that SOCS1 can be induced by the activated janus kinase family (JAK) and the signaling transduction and activators of transcription family (STATs), and may downregulate the JAK/Stats signaling pathway (8), which plays an important role in the initiation and activation of inflammation, through inhibition of the phosphorylation of kinase mentioned above.

Recently, Serezani et al found that expression of myeloid differentiation factor (MyD88) (9), a downstream signaling molecule of the Toll-like receptor (TLR) 4 pathway $(10,11)$, and responsiveness to the TLR4 ligand LPS were decreased by Statl siRNA silencing in macrophages and restored by Socs 1 siRNA. The overexpression of TLR4 was found to induce excess inflammation, which is involved in tissue injury and epithelial damage. Our previous study demonstrated that TLR4 plays an important part in airway inflammation, particularly in alveolitis. We stimulated the alveolar epithelium with different concentrations of LPS and found that the level of mRNA of TLR4 in type II alveolar epithelium increased in accordance with the concentration of LPS (12).

Idiopathic pulmonary fibrosis (IPF) is a progressive fibrotic disease of the lungs that increases with advanced age. Recent evidence indicates that TLRs are implicated in the pathogenesis of fibrotic lung disorders $(13,14)$. However, it remains unknown whether SOCS1 is a repressor factor for the chronic inflammation of IPF through its inhibitory effect on the TLR signaling pathway.

In our previous study, we hypothesized that downregulation of SOCS1 suppresses chronic inflammation of the airway, which was initiated by the TLR4-related signaling pathway. Therefore, we conducted a recombinant lentiviral vector with a knockdown effect targeting SOCS1, using an RNA interference method. The aim of the present study was to further investigate the regulatory effect of SOCS1 on alveolar epithelial cells. 


\section{Materials and methods}

Materials and reagents. MTT kit was purchased from SigmaAldrich Corp. (St. Louis, MO, USA). DMEM medium was provided by Sigma-Aldrich. Fetal bovine serum (FBS) was obtained from Invitrogen Gibco (NY, USA). Packaging cell 293T was purchased from the Cytology Center of the Chinese Academy of Science (Shanghai, China). Recombinant human TGF- $\beta 1$ was purchased from R\&D Systems (Minneapolis, MN, USA). The restricted enzyme was purchased from Fermentas (Hanover, MD, USA), and Protease Inhibitor Cocktail was from Merck Calbiochem (Darmstadt, Germany). Rabbit polyclonal antibody against human SOCS1 was from Santa Cruz Biotechnology (Heidelberg, Germany), and against human $\beta$-actin was from Sigma-Aldrich. The gel extraction kit was from Qiagen (Hilden, Germany). Oligonucleotides were synthesized by Biotelevector (Shanghai, China).

Cell lines and culture conditions. A549 cells (ATCC CL-185) were preserved at the Cytology Center of the Chinese Academy of Science. Cells were maintained in DMEM medium supplemented with $10 \% \mathrm{FBS}$ at $37^{\circ} \mathrm{C}$ under an atmosphere of $5 \% \mathrm{CO}_{2}$. The media were changed every $48 \mathrm{~h}$.

In order to examine cell survival, cell cultures at $\sim 70 \%$ confluence were serum-starved overnight and then treated with TGF- $\beta 1$ in culture medium containing $2 \%$ FBS for $72 \mathrm{~h}$.

Construction of RNA interference plasmids. Three complementary oligonucleotides necessary to create the hairpin insert for pPlasRi cloning vectors were designed. The sequences of the oligonucleotides were as follows: SOCS1 RNA interference short hairpin (sh) insert sequences SH1: 5'-TGG TTG TTG TAG CAG CTT AAC TTT CAA GAG AAG TTA AGC TGC TAC AAC AAC CTT TTT TC-3' and 5'-TCG AGA AAA AAG GTT GTT GTA GCA GCT TAA CTT CTC TTG AAA GTT AAG CTG CTA CAA CAA CCA-3'; SH2: 5'-TGC ACC TCC TAC CTC TTC ATG TTT CAA GAG AAC ATG AAG AGG TAG GAG GTG CTT TTT TC-3' and 5'-TCG AGA AAA AAG CAC CTC CTA CCT CTT CAT GTT CTC TTG AAA CAT GAA GAG GTA GGA GGT GCA-3'; SH3: 5'-TGA CAA TGC AGT CTC CAC AGC ATT CAA GAG ATG CTG TGG AGA CTG CAT TGT CTT TTT TC-3' and 5'-TCG AGA AAA AAG ACA ATG CAG TCT CCA CAG CAT CTC TTG AAT GCT GTG GAG ACT GCA TTG TCA-3'. BbsI and XhoI restriction sites were introduced at the end. A pair of oligonucleotides was annealed according to the following method: $95^{\circ} \mathrm{C}, 5 \mathrm{~min} ; 85^{\circ} \mathrm{C}, 5 \mathrm{~min}$; $75^{\circ} \mathrm{C}, 5 \mathrm{~min} ; 70^{\circ} \mathrm{C}, 5 \mathrm{~min}$. After annealing, double-stranded shRNA templates were diluted (1:50) and further ligated to the lentiviral vector pP113.7, which was double digested with XhoI/HpaI. Vector Pll3.7 was used without oligonucleotides as a negative control. JM $109 E$. coli cells were then transformed; we selected the colony and extracted the recombinant plasmids for sequencing.

Lentiviral packaging and titering. We co-transfected 293T cell lines with each recombinant interfering plasmid, which was proved by sequencing, together with the other three types of packaging plasmids (pRsv-REV, pMDlg-pRRE and pMD2G, respectively). After $72 \mathrm{~h}$, the supernatant was harvested and concentrated. The virus titer was determined and calibrated in the $293 \mathrm{~T}$ cell lines. The $293 \mathrm{~T}$ cell culture medium was then filtered and concentrated to obtain a SOCS siRNA lentivirus solution and kept in a collection cup at $-80^{\circ} \mathrm{C}$.

Lentiviral transfection in vitro. To screen the target sequence with the highest interference efficacy in vitro, A549 cells were plated in 6-well plates $18-24 \mathrm{~h}$ prior to the experiment and serum-starved overnight, by which time the cells had reached $60-75 \%$ confluence. The supernatant from three lentiviruses (100 $\mu \mathrm{l})$ was added to the wells, according to the following groups: group A, blank; group $\mathrm{B}$, negative control lentivirus plasmid; group $\mathrm{C}, \mathrm{SH} 1$; group $\mathrm{D}, \mathrm{SH} 2$; group $\mathrm{E}, \mathrm{SH}$. The transfection medium was replaced at $8 \mathrm{~h}$ with fresh growth medium and cells were further incubated for $24 \mathrm{~h}$, or up to $72 \mathrm{~h}$ in order to conduct further Western blot analysis.

Fluorescence microscopy. Following 24-h transfection, we observed the transfection effect under a fluorescence microscope. After $72 \mathrm{~h}$, the cells were harvested and Western blotting was used to analyze the extracted protein. Subsequently, the best target site with the highest interfering efficiency was identified.

SDS-PAGE and Western blotting. To screen the target site, $10^{6}-10^{7}$ cells were disrupted in ice-cold cell lysis buffer. Protein sample concentrations were determined using a standard protein concentration assay (Bio-Rad, Herts, UK). Total protein $(20 \mu \mathrm{g})$ from each sample was loaded onto $10 \%$ polyacrylamide gels (Bio-Rad). After electrophoresis, separated proteins were transferred onto immunoblot polyvinylidene fluoride membranes (Merck Millipore, USA). Membranes were then blocked with $5 \%$ bovine serum albumin (BSA) in TBST for $1 \mathrm{~h}$ at room temperature. Primary antibodies $(1: 10,000$ for $\beta$-actin, 1:1,000 for SOCS1 antibodies) were added to Blotto or $0.1 \%$ BSA in TBST and incubated with membranes overnight at $4^{\circ} \mathrm{C}$ on a rocking platform. After washing with TBST repeatedly (three times, $15 \mathrm{~min}$ each), membranes were probed with corresponding anti-rabbit horseradish peroxidase (HRP)conjugated secondary antibody for $2 \mathrm{~h}$ at room temperature. Membranes went to a second stage of washes in TBST, three times each for $15 \mathrm{~min}$. Immunoblots were visualised by enhanced chemiluminescence (GE Healthcare, Chalfont St. Giles, UK). Results were normalized against $\beta$-actin band density used as a loading control.

In vitro cell growth assay. The A549 cells and SOCS1 knockdown A549 cells were placed in 96-well plates, $100 \mu \mathrm{l}$ $(5,000$ cells/well $)$ in each well, and treated with TGF- $\beta 1$ ( $2 \mathrm{ng} / \mathrm{ml}$ ); each group was plated in 8 wells. The inhibitory rates of the drugs in the groups treated after TGF- $\beta 1$ with or without dexamethasone (DXM) were calculated after $48 \mathrm{~h}$ of incubation. Control cells received only media. The cells were incubated at $37^{\circ} \mathrm{C}$ in an atmosphere of $5 \% \mathrm{CO}_{2}$ for at least $72 \mathrm{~h}$. Four hours before the end of incubation, $100 \mu \mathrm{g}$ of MTT was added to each well. At the end of the incubation period, the MTT was removed and $100 \mu 1$ isopropanol mixed with concentrated $\mathrm{HCl}$ was then added to terminate the reaction. The optical density (OD) of each well was measured using a microculture plate reader with a test wavelength of $570 \mathrm{~nm}$. 


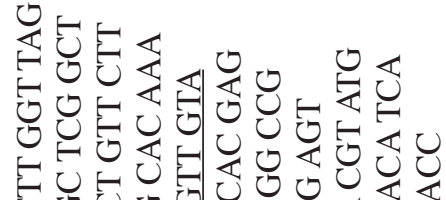

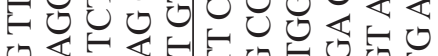

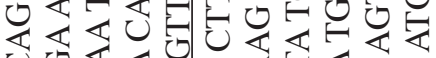

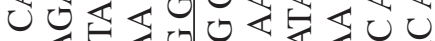

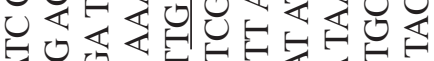

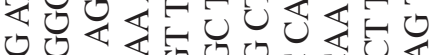
Uि Oण

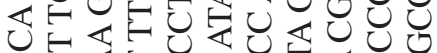

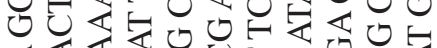

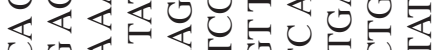

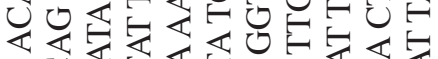
OU OU

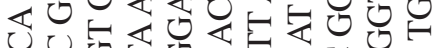
U

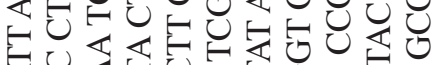

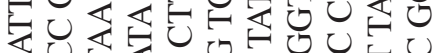
EU O OU U

플

$\Xi$

总

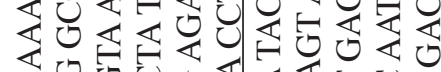
U

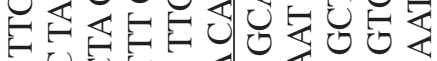

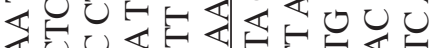

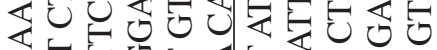

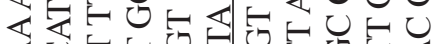

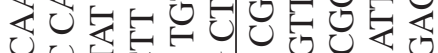
UU

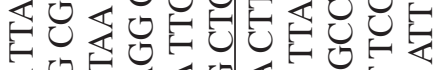
《O

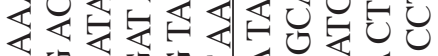

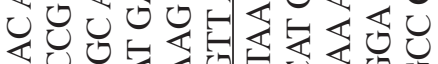

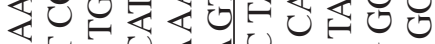

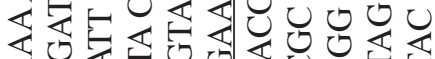
$\varangle<$ o

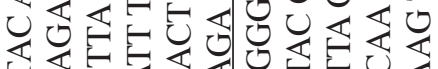

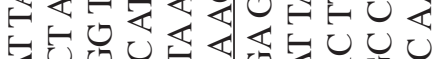

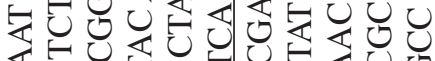
o o o 01 o

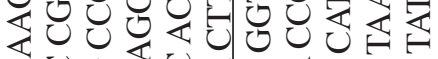
$\varangle 0 \ll$

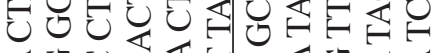

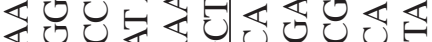

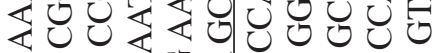

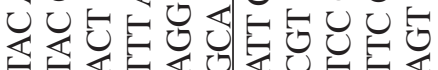

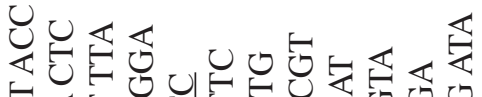

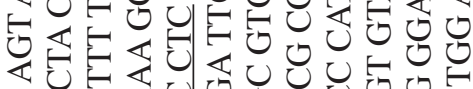

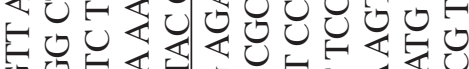
0

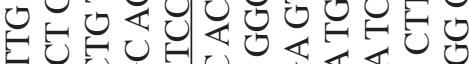

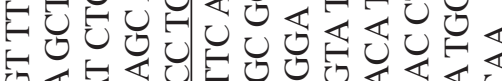

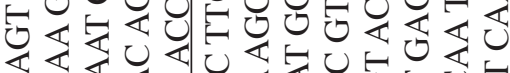

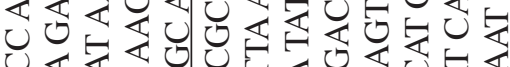

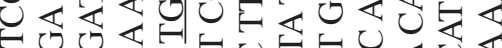

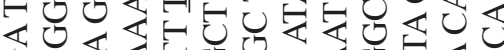
OU

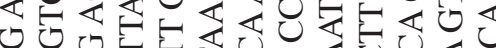
U U U.

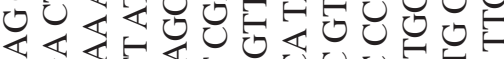
U

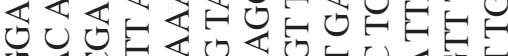

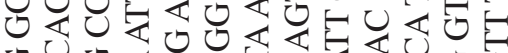

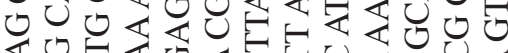
Ư

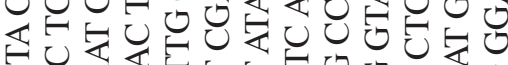

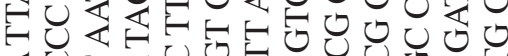
《UE

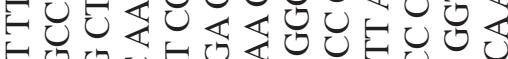
EO O 0 O o O

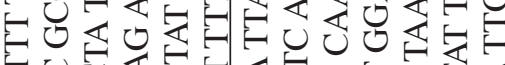

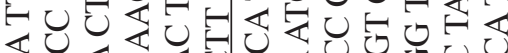

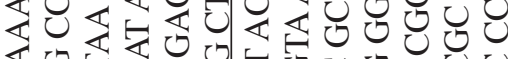

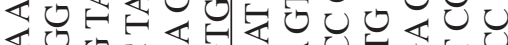

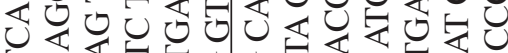

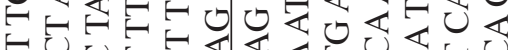

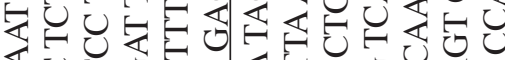
xum 0 .

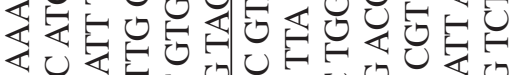
3u<

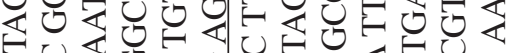

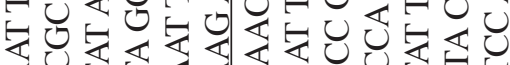

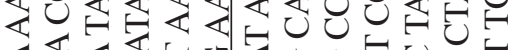

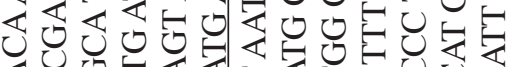

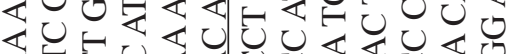

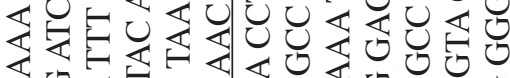

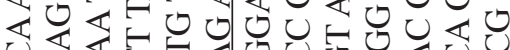

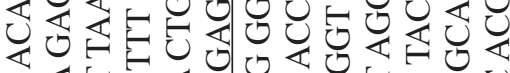

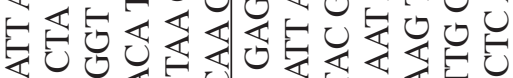
Thu So xu

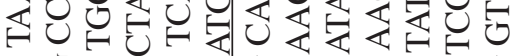

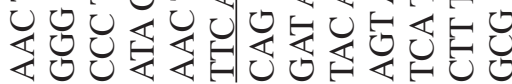

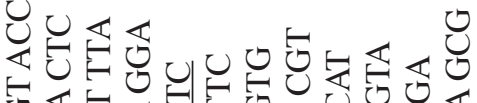

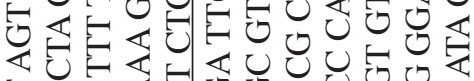

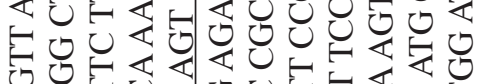

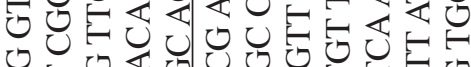

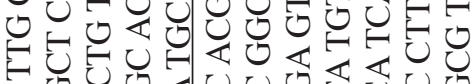

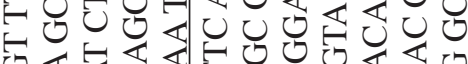

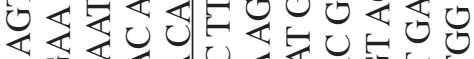
U.

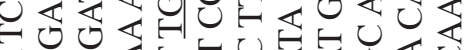

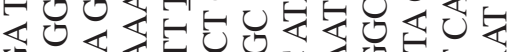
ơ O SEx o

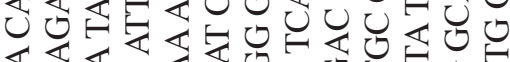

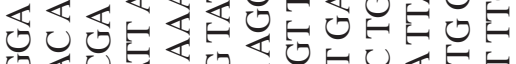

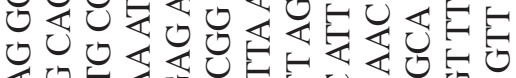

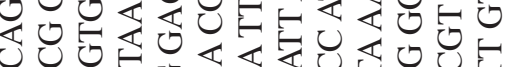
列

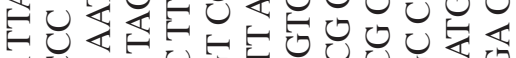

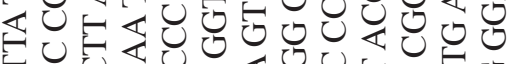
E

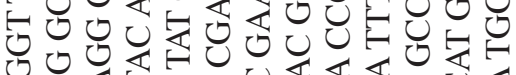
o

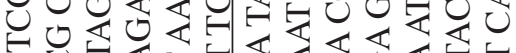

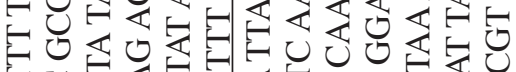
Futrustrut

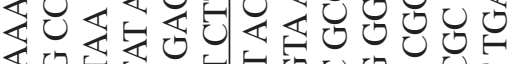
年

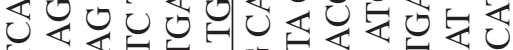

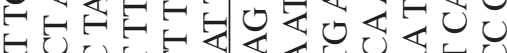

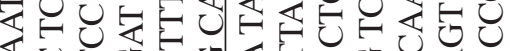

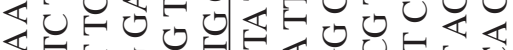

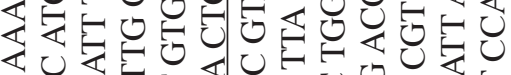

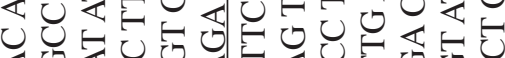

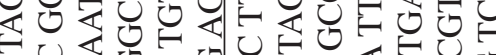

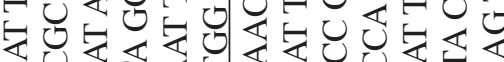

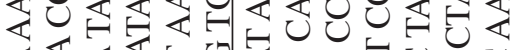

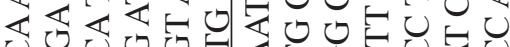
U.

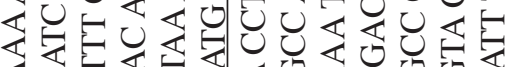

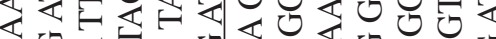
\$

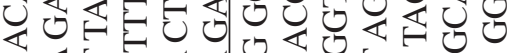

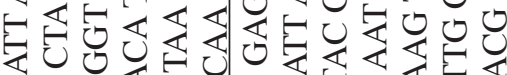
rey y y

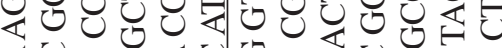

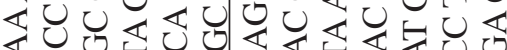

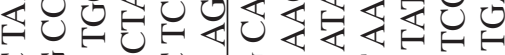

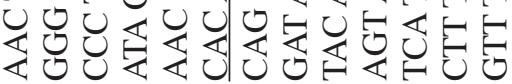




\section{Results}

Sequencing results of recombinant RNA interference plasmids. As shown in Table I, the sequencing results of the three recombinant plasmids completely matched the designed sequences, and the detailed sequencing could be seen as the supplement material.

SOCS1 RNA interference lentivirus particles and titering. Forty-eight hours after co-transfection of the recombinant lentiviral vector Plentivirus-SOCS1-siRNA (Fig. 1), the supernatant was filtered and concentrated. The lentiviral stock was diluted and titered in A549 cells. The titering concentrations of three recombinant lentiviruses were as follows: $\mathrm{SH} 1,1.11 \times 10^{7} \mathrm{TU} / \mathrm{ml}$; $\mathrm{SH} 2,1.13 \times 10^{7} \mathrm{TU} / \mathrm{ml}$; SH3, 1.26×107 $\mathrm{TU} / \mathrm{ml}$.

Screening of target site with the optimal interference efficacy. Western blot analysis was used to screen the optimal target interference site, and target $\mathrm{SH} 2$ showed the highest inhibition efficacy. This group of recombinant lentiviruses was used for further assay of cell survival. (Fig. 2)

MTT assay. MTT assay results showed that A549 cells transfected by Plentivirus-siSOCS1 (the knockdown group), exhibited strong inhibition of cell proliferation when compared to the control and the negative control groups (Fig. 3).

\section{Discussion}

SOCS was discovered in 1997 and was confirmed by three different laboratories (15). To date, eight members have been identified. Among these, SOCS1 and SOCS3 are recognized to have the greatest downregulatory activity on the signaling pathway (16). To the best of our knowledge, this is the first attempt to demonstrate the effect of SOCS1 on the apoptosis of alveolar epithelial cells. We observed an interference knockdown effect of the A549 cells on target protein, which was infected with the lentiviral recombinant vector. The sequencing results confirm the correct sequences of the oligonucleotides, which were inserted into the interference plasmids. Regarding the interference efficacy, the sequencing target $\mathrm{SH} 2$ demonstrated the highest inhibitory effect, which was confirmed by Western blotting. This cell line, which stably exhibited an inhibitory effect on SOCS1, was used for the study on cell apoptosis.

Assuming that SOCS1 effectively interferes with a target site, we constructed a recombinant lentiviral vector for RNA interference targeting the SOCS1 gene, which was then transfected into alveolar epithelial A549 cells, with packaging plasmids as well, in order to observe its effect on SOCS1 gene expression and cell proliferation. Our results showed that the recombinant lenvivirus-SOCS1-siRNA virus particle inhibited SOCS1 gene expression effectively in the A549 cell line. Cell lines infected with the RNA interference virus exhibited lower proliferation than that of the control and negative control groups $(\mathrm{P}<0.01)$. The lentiviral particles for RNA interference, targeting the SOCS1 gene, specifically suppressed SOCS1 gene expression and inhibited alveolar epithelial cell growth and proliferation.

In principle, there are two general siRNA delivery methods: one uses chemically synthesized 21-23 bp siRNA
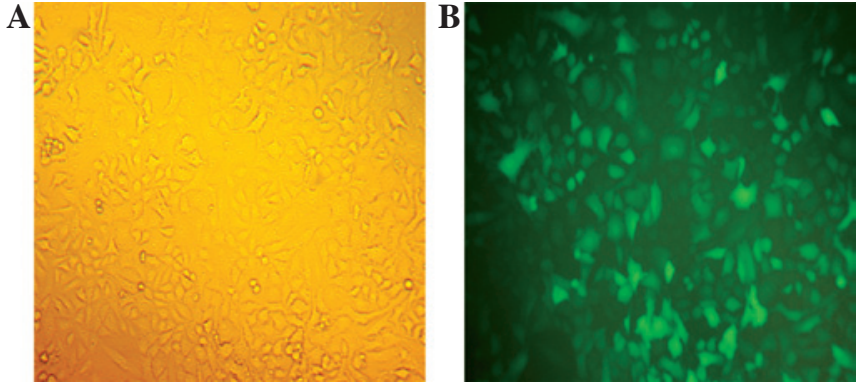

Figure 1. (A) Cell morphology was noted under light microscopy. (B) Most A549 cells were infected by the lentiviral vector $(>90 \%)$ and were stained with green fluorescence protein as determined by fluorescence microscopy.

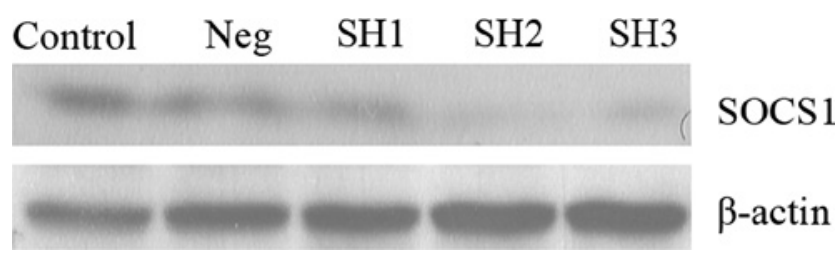

Figure 2. Expression of target protein using Western blotting. Upper panel, SOCS1; lower panel, $\beta$-actin. Groups: Control, A549 cells without vector; Neg, empty plamid; $\mathrm{SH} 1$, lentivirus target $\mathrm{SH}$; $\mathrm{SH}$ 2, lentivirus target $\mathrm{SH}$; $\mathrm{SH} 3$, lentivirus target SH3.

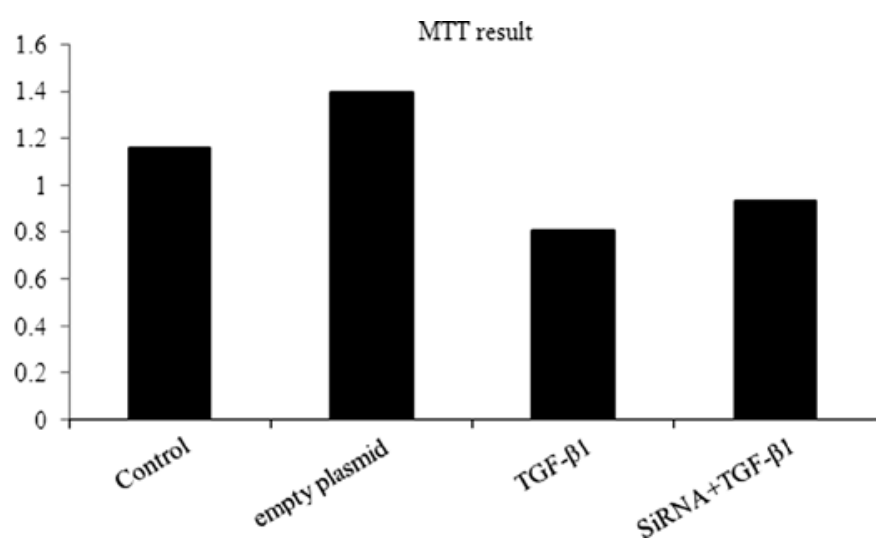

Figure 3. MTT results. Control, A549 cells without stimulation of TGF- $\beta 1$; Empty plasmid, A549 cells transfected with empty plasmid, no stimulation of TGF- $\beta 1$; TGF- $\beta 1$, A549 cells stimulated with TGF- $\beta 1$; siRNA+TGF- $\beta 1$, SOCS1-knockdown A549 cells with stimulation of TGF- $\beta 1$.

with temporary interference effect, while the other approach is a vector-based siRNA method, that includes the use of interference plasmid and packaging plasmids, which exhibit stable and long-time knockdown activity of targeting genes. In recent years, research has focused on the use of lentivectors, which - like their simple retrovirus counterparts - are devoid of viral proteins, free from replication competent virus, and are additionally able to transduce non-dividing cells (17). There have been three generations of lentiviral particles. The lentiviral particle used in our study belongs to the third generation. It consisted of three plasmids, pRsv-REV, pMDlg-pRRE, pMD2G, and the interference plasmid. Among them, the interference plasmid contains a region of green fluorescence protein, which could easily identify the transfection efficacy. 
Our results showed that there were significant RNA interference effects on SOCS1 in alveolar epithelial cells after RNA interference expression plasmid transfection.

IPF is a chronic, interstitial lung disease, characterized by diffuse alveolar damage and severe fibrosis, resulting in a steady deterioration of lung function and gas exchange. Epithelial cell death is a crucial early step in the development of the disease (18), followed only later by the fibrotic stage. IPF is triggered by alveolar basement membrane disruption and, in the presence of persisting injurious pathways (19), activated TGF- $\beta$ leads to enhanced epithelial apoptosis and epithelial-to-mesenchymal transition as well as fibroblast $(20,21)$ transformation into myofibroblasts which are resistant to apoptosis $(22,23)$. In the present study, alveolar epithelial cell apoptosis was measured using MTT assay. The RNA interference group demonstrated a significantly high level of cell death compared to the control group, which indicated that downregulation of the SOCS1 gene with RNA interference may promote the apoptosis of alveolar epithelial cells and may further lead to the pathogenesis of IPF.

These results advance a novel targeting site for the treatment of idiopathic pulmonary fibrosis. In particular, our findings demonstrating that the lenvivirus-SOCS1-siRNA recombinant virus particle effectively knocks down SOCS1 gene expression in alveolar epithelial cells, will greatly benefit further research on the function of SOCS1. Furthermore, it may be used for animal models of IPF.

In conclusion, we demonstrated a stable cell line of alveolar epithelium using RNA interference targeting SOCS1. MTT assay showed that SOCS1 knockdown promotes the apoptosis of alveolar epithelial cells.

\section{References}

1. Lexander WS and Hilton DJ: The role of suppressors of cytokine signaling (SOCS) proteins in regulation of the immune response. Annu Rev Immunol 22: 503-529, 2004.

2. Yoshimura A, Naka T and Kubo M: SOCS proteins, cytokine signalling and immune regulation. Nat Rev Immunol 7: 454-465, 2007.

3. Kubo M, Hanada T and Yoshimura A: Suppressors of cytokine signaling and immunity. Nat Immunol 4: 1169-1176, 2003.

4. Nakagawa R, Naka T, Tsutsui H, et al: SOCS-1 participates in negative regulation of LPS responses. Immunity 17: 677-687, 2002.

5. Baetz A, Frey M, Heeg K, et al: Suppressor of cytokine signaling (SOCS) proteins indirectly regulate toll-like receptor signaling in innate immune cells. J Biol Chem 279: 54708-54715, 2004.
6. Mansell A, Smith R, Doyle SL, et al: Suppressor of cytokine signaling 1 negatively regulates Toll-like receptor signaling by mediating Mal degradation. Nat Immunol 7: 148-155, 2006.

7. Qing Y, Costa-Pereira AP, Watling D, et al: Role of tyrosine 441 of interferon- $\gamma$ receptor subunit 1 in SOCS-1-mediated attenuation of STAT1 activation. J Biol Chem 280: 1849-1853, 2005.

8. Fenner JE, Starr R, Cornish AL, et al: Suppressor of cytokine signaling 1 regulates the immune response to infection by a unique inhibition of type I interferon activity. Nat Immunol 7: 33-39, 2006.

9. Serezani CH, Lewis C, Jancar S, et al: Leukotriene B4 amplifies NF- $\kappa \mathrm{B}$ activation in mouse macrophages by reducing SOCS1 inhibition of MyD88 expression. J Clin Invest 121: 671-682, 2011.

10. Ryo A, Suizu F, Yoshida Y, et al: Regulation of $\mathrm{NF}-\kappa \mathrm{B}$ signaling by Pin1-dependent prolyl isomerization and ubiquitin-mediated proteolysis of p65/RelA. Mol Cell 12: 1413-1426, 2003.

11. Baetz A, Koelsche C, Strebovsky J, et al: Identification of a nuclear localization signal in suppressor of cytokine signaling 1. FASEB J 22: 4296-4305, 2008

12. Zhou M, Wan HY, Huang SG, et al: Expression of Toll-like receptor 4 in human alveolar epithelial cells and its role in cellular inflammation. Natl Med J Chin 88: 2112-2116, 2008.

13. Margaritopoulos GA, Antoniou KM, Karagiannis K, et al: Investigation of Toll-like receptors in the pathogenesis of fibrotic and granulomatous disorders: a bronchoalveolar lavage study. Fibrogenesis Tissue Repair 3: 20-28, 2010.

14. Trujillo G, Meneghin A, Flaherty KR, et al: TLR9 differentiates rapidly from slowly progressing forms of idiopathic pulmonary fibrosis. Sci Transl Med 2: 57ra82, 2010.

15. Starr R, Willson AT, Viney EM, et al: A family of cytokine inducible inhibitors of signaling. Nature 387: 917-921, 1997.

16. Kinjyo I, Hanada T, Inagaki-Ohara K, et al: SOCS1/JAB is a negative regulator of LPS-induced macrophage activation. Immunity 17: 583-591, 2002

17. Escors D and Breckpot K: Lentiviral vectors in gene therapy: their current status and future potential. Arch Immunol Ther Exp 58: 107-119, 2010.

18. Mercer PF, Johns RH, Scotton CJ, et al: Pulmonary epithelium is a prominent source of proteinase-activated receptor-1-inducible CCL2 in pulmonary fibrosis.Am J Respir Crit Care Med 179: 414-425, 2009.

19. Harada T, Nabeshima K, Hamasaki M, et al: Epithelialmesenchymal transition in human lungs with usual interstitial pneumonia: quantitative immunohistochemistry. Pathol Int 60: 14-21, 2010

20. Drakopanagiotakis F, Xifteri A, Polychronopoulos V, et al: Apoptosis in lung injury and fibrosis. Eur Respir J 32: 1631-1638, 2008.

21. Yu H, Königshoff M, Jayachandran A, et al: Transgelin is a direct target of TGF-beta/Smad3-dependent epithelial cell migration in lung fibrosis. FASEB J 22: 1778-1789, 2008.

22. Zhong Q, Zhou B and Ann DK: Role of ER stress in EMT of alveolar epithelial cells: effects of misfolded surfactant protein. Am J Respir Cell Mol Biol 12: 17-23, 2010.

23. Sisson TH, Mendez M, Choi K, et al: Targeted injury of type II alveolar epithelial cells induces pulmonary fibrosis. Am J Respir Crit Care Med 181: 254-263, 2010 